\title{
Preparation and Structural Investigation of PMMA-Polystyrene 'Janus Beads' by Rapid Evaporation of an Ethyl Acetate Aqueous Emulsion
}

\author{
Yuji Kiyono \\ Graduate School of Photonic Science, Chitose Institute of Science and Technology, Chitose 066-8655, Japan \\ Laszlo Szikszai and Junichi Watanabe \\ Department of Bio-and Material Photonics, Chitose Institute of Science and Technology, Chitose 066-8655, Japan \\ Olaf Karthaus* \\ Graduate School of Photonic Science, and Department of Bio-and Material Photonics, \\ Chitose Institute of Science and Technology, Chitose 066-8655, Japan
}

\author{
Roland Hass, Martin Maiwald, Oliver Reich, and Hans-Gerd Löhmannsröben \\ innoFSPEC, Department for Chemistry/Physical Chemistry, \\ University of Potsdam, 14476 Potsdam-Golm, Germany
}

(Received 9 December 2011; Accepted 4 May 2012; Published 21 July 2012)

\begin{abstract}
In this paper, the formation of microscopic polystyrene/poly(methyl methacrylate) Janus particles by using environmentally benign solvents and surface active agents is presented. Janus particles are formed by the rapid polymer phase separation that occurs when an oil-in-water suspension is allowed to dry. Biodegradable or bio-inert organic solvents and surface active agents are used. These solvents affect the particle morphology, which is studied in detail. The environmentally friendly solvent ethyl acetate can replace the more commonly used toluene for the preparation of Janus particles. Confocal Raman microscopy is used to visualize and analyze the phase separated Janus particles. Results indicate that the polystyrene hemisphere as minor component within the particle is covered with a thin PMMA layer on its surface. [DOI: 10.1380/ejssnt.2012.360]
\end{abstract}

Keywords: Surface structure, morphology, roughness, and topography; Raman scattering spectroscopy; Photoluminescence; polymer; phase separation

\section{INTRODUCTION}

In 1989, Casagrande et al. described the preparation of microscopic glass beads with two hemispheres having different surface properties [1]. Especially the expected unique interactions with liquid-liquid interfaces triggered research on those 'Janus particles' $[2,3]$. It has been reported that such hydrophilic-hydrophobic particles can be used to stabilize emulsions of immiscible liquids by the Pickering effect [4]. In addition, there are many other possible applications for phase separated microscopic particles with two hemispheres that have different properties, for example in biosensors [5] or electronic paper [6]. There are several routes to produce such heterogeneous particles, and for polymer particles the main established route is the multistage polymerization with added seed particles [7]. The morphology of the phase separated particles depends on a delicate interplay between the interfacial free energy (reached at thermodynamic equilibrium) and the viscosity change during polymerization (kinetic and non-equilibrium) [8]. Additives can be used to control morphology of such composite particles [9]. In such a multidimensional coordinate system with many independent variables, particles with spherical as well as nonspherical shape and smooth or rough surface morphologies can be prepared [10].

A more direct method for the preparation of Janus polymer particles is the mixing of two or more polymers that are dissolved in a common organic solvent, with water.

*Corresponding author: kart@photon.chitose.ac.jp
On the one hand, this method requires the additional step of polymerizing the monomer in a homogeneous solution before particle preparation, but on the other hand, by using a polymer, the nonlinear effects in an emulsion where the simultaneous phase separation of the polymer influences the polymerization kinetics and thus the polymer molecular weight and its distribution are avoided. An oil-in-water (o/w) emulsion containing droplets of two polymers dissolved in an organic solvent leads to phaseseparated polymer particles upon the evaporation of the organic solvent. A polymer system that has been investigated in detail is the polystyrene/poly(methyl methacrylate)/ toluene/surfactant $\mathrm{o} / \mathrm{w}$ emulsion [11-13]. In these systems, the molecular weight of the polymer, the kind of organic solvent, and the surfactant play a role in determining the shape of the Janus particles. In many publications toluene and sodium dodecyl sulfate (SDS) are used as solvent and surfactant, respectively.

For a large scale production of these potentially useful Janus particles, a more environmentally friendly and carbon neutral methodology has to be established.

In this paper, solvents other than toluene and biodegradable or bio-inert surfactants are used for the preparation of Janus beads. The particles are characterized by optical and electron microscopy. The surface topography of the phase-separated particles was imaged with scanning electron microscopy.

Even though PS and PMMA have different refractive indices, and Janus beads can be imaged by means of optical microscopy, the optical contrast is limited. We have already reported on the doping of PS/PMMA blends with the electron acceptor tetracyanoquinodimethane (TCNQ). PS forms a charge-transfer complex with TCNQ 


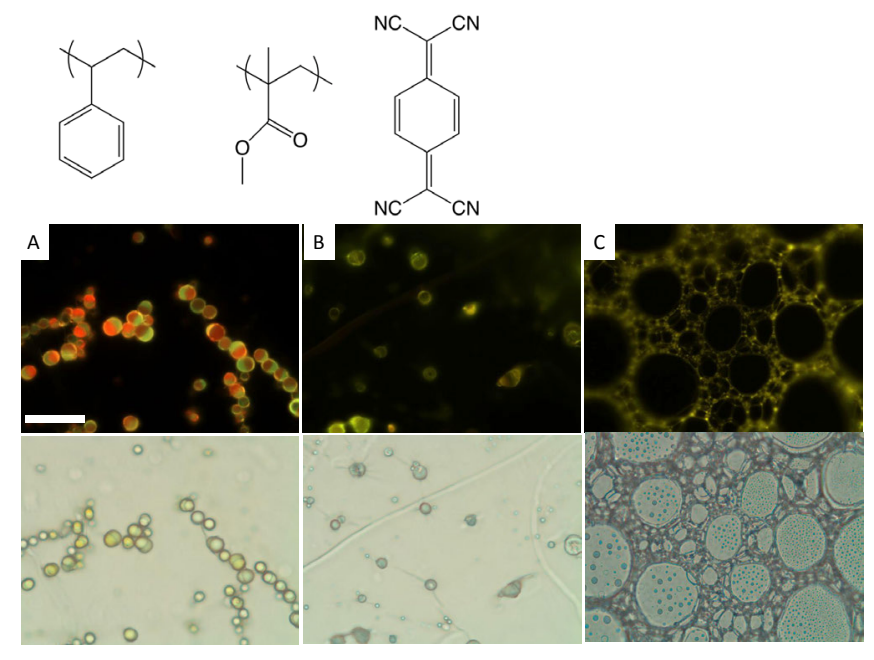

FIG. 1: Chemical structure of the two polymer, polystyrene and poly(methylmethacrylate), and the dopant TCNQ that exhibits different fluorescence colors in polystyrene (red fluorescence) and PMMA (green fluorecence). Fluorescence (top) and transmission (bottom) microscope images of structures obtained from emulsions using different organic solvents. The organic solvent contains $1.5 \mathrm{mg} / \mathrm{mL}$ of PS and $1.5 \mathrm{mg} / \mathrm{mL}$ PMMA The aqueous phase contains $0.12 \mathrm{wt} \%$ poly(vinylalcohol) as emulsifier. A: ethyl acetate; B: toluene; C: geranyl formate. The scale bar is $50 \mu \mathrm{m}$.

that shows orange fluorescence, while TCNQ embedded in PMMA emits green fluorescence upon excitation in the blue spectral range [14]. Thus fluorescence microscopy is sensitive to the individual polymer (Fig. 1).

As an alternative, confocal Raman microscopy is a wellsuited nondestructive optical method to generate images with a spatial resolution in the sub-micrometer range e.g. to visualize phase separated structures [15]. One advantage of this method is the direct access of chemical information of the sample under study without the use of contrasting agents as indicator. Additionally, a confocal arrangement allows a 3 -dimensional imaging by using $\mathrm{z}$ scan tomography.

\section{EXPERIMENTAL}

PMMA (Mw: $120,000 \mathrm{~g} / \mathrm{mol}), \quad$ PS (Mw: $280,000 \mathrm{~g} / \mathrm{mol}$ ), chitosan (low molecular weight and medium molecular weight), poly(vinyl alcohol) (Mw: 31,000-50,000 g/mol, 98-99\% hydrolyzed) and geranyl formate were from Aldrich, Inc. Glucomannan (commercial name 'Rheolex', viscosity of a $1 \%$ solution: $34,600 \mathrm{mPa}$ s at $25^{\circ} \mathrm{C}$ ) was from Shimizu Chemical Corp., Japan. Pectin (from Citrus, 50-70\% galacturonic acid), hydroxypropyl cellulose viscosity of a $2 \%$ solution: 2.0-2.9 $\mathrm{mPa} \mathrm{s}$ at $20^{\circ} \mathrm{C}$, poly(vinylpyrrolidone) (K30), toluene, and ethyl acetate were from Wako Pure Chemical Industries Ltd., Japan. TCNQ was purchased from TCI, Japan.

Scanning electron microscope images were taken with a Keyence VE-8800 microscope, optical and fluorescence microscope images (excitation: 400-440 nm) were taken with an Olympus IX-51 microscope equipped with a
SPOT-RT CCD camera. For the Raman experiments a confocal microscopic setup alpha300 R (WITec Wissenschaftliche Instrumente und Technologie GmbH, Germany) including a continuous wave operating laser with an excitation wavelength at $\lambda_{\mathrm{ex}}=532 \mathrm{~nm}$ was used. A microscope objective (Olympus MPlan FL N) with a magnification of 100 and a numerical aperture of $N A=0.9$ focuses the laser beam onto the sample. The resulting excitation spot size corresponds to an Airy disc diameter of $d_{\text {Airy }}=1.22 \times \lambda_{\text {ex }} / N A=0.72 \mu \mathrm{m}$. For all Raman measurements the excitation power was $5 \mathrm{~mW}$ at sample and the integration time was $1 \mathrm{~s}$ for a single spectrum which corresponds to one pixel in the 2-D Raman images. The spatial step size for the Raman scans was $0.1 \mu \mathrm{m}$ in all directions.

The generated photons were collected with the same microscope objective in a $180^{\circ}$-backscattering geometry. A Raman edge filter blocks the anti-Stokes shifted light and the laser light. The Stokes-shifted light passes the edge filter and is coupled into a collection fiber that acts as a pinhole for the confocal arrangement. To reach a high axial confocality in the setup and remain a sufficient amount of collected Raman scattered photons a fiber with a core diameter of $d_{\text {core }}=50 \mu \mathrm{m}$ was chosen [16]. The spatial resolution in air can be estimated to $d_{x y} \approx 0.24 \mu \mathrm{m}$ and the axial resolution to $d z \approx 0.92 \mu \mathrm{m}$ [17]. Raman spectra of a Si-substrate were measured to verify the calculated axial resolution. For each Raman spectrum the focal spot was moved along the optical axis $z$ inside and outside the Si-substrate with a step size of $0.2 \mu \mathrm{m}$. The Raman signal of $\mathrm{Si}$ at $521 \mathrm{~cm}^{-1}$ versus the relative position $z$ was plotted and shows a full width at half maximum (FWHM) of $0.95 \mu \mathrm{m}$.

The collected Raman photons are launched into a spectrograph (UHT S300, WITec GmbH) with a spectral resolution of $8 \mathrm{~cm}^{-1}$. A back illuminated, thermo-electric (TE) cooled CCD (iDUS 401, $1024 \times 127$ pixel, Andor Technology) operating at $T_{\mathrm{CCD}}=-65^{\circ} \mathrm{C}$ was used to detect the Raman signals. A more detailed description of such a confocal Raman microscope is given in [16]. Oilin-water emulsions were prepared by placing $0.75 \mathrm{~mL}$ oil phase and $2 \mathrm{~mL}$ water phase in a $6 \mathrm{ml}$ sample container that was shaken for $10 \mathrm{~s}$ using an orbital shaker (Lab Dancer S25, Ika Corp., Germany). For fluorescence microscopic imaging, the oil phase contained 100 ppm TCNQ as a fluorescent dopant.

For observation, one drop of the emulsion was cast on a glass slide (for optical and electron microscopy), or on a Si-wafer (for Raman microscopy) and the emulsion was allowed to dry.

\section{RESULTS AND DISCUSSIONS}

The o/w emulsions were cast on microscope glass slides and allowed to dry. First, the organic solvent evaporated in the course of a few minutes, and the water dried up within one to two hours. It is also possible to microscopically investigate the polymer particles while the water is still present. Figure 1 shows the samples prepared from three different solvents. Ethyl acetate was chosen, because it has a very low toxicity [18] with an LD50 as high as $11.3 \mathrm{~g} / \mathrm{kg}$. Its medium boiling point $\left(71^{\circ} \mathrm{C}\right)$, low 
enthalpy of evaporation $(31.94 \mathrm{~kJ} / \mathrm{mol})$, and high vapor pressure $\left(80 \mathrm{~mm} \mathrm{Hg}\right.$ at $\left.20^{\circ} \mathrm{C}\right)$ allows for the rapid evaporation which is necessary for the 'freezing' of the dynamic phase separation structure of both polymers. The second solvent was toluene, which is also a good solvent for both polymers. It has been used for the preparation of phase separated Janus beads in the past $[11,12]$. Even though toluene has a higher boiling point than water, its low enthalpy of evaporation still leads to a reasonably fast evaporation to form polymer microbeads in a water emulsion. Drawbacks of toluene are its toxicity and low biodegradability, thus making it necessary to capture and recycle the toluene vapor if this technique should be used on an industrial scale. The third solvent to show the influence of evaporation rate should have an even higher boiling point than toluene and geranyl formate with a boiling point of $216{ }^{\circ} \mathrm{C}$ was selected. In this case, biodegradability is guaranteed, because after being released into the environment, it will decompose to geraniol, a natural alcohol, and formic acid.

In the following we will explain how the variation of several parameters influences the formation of Janus beads.

\section{A. Effect of solvent}

The effect of the organic solvent in a series of experiments by using poly(vinyl alcohol) as emulsifier was investigated and is presented in Fig. 1. A polymer mixture in ethyl acetate (Fig. 1A) forms beads in which both polymers are present in the same bead. In addition the beads clearly show a phase separated structure, forming 'true' Janus beads with symmetrical hemispheres of the two polymers with a planar interface between them. Since the orientation of the beads on the glass substrate is random, only few of the beads will be oriented in such a way that the two hemispheres can be seen clearly. Since the ethyl acetate evaporates faster than water, the organic phase diminishes and the aqueous phase is the continuous phase at all times-beads are formed. The phase separated structure within each bead is nearly invisible in the optical transmission micrographs, but identification of the two polymers was achieved by fluorescence microscopy. Even though the Janus beads are not strictly monodisperse, the size distribution is moderate. The beads shown in the fluorescence micrograph show a polydispersity of around two.

Using toluene as a solvent also leads to polymer beads, as can be seen in Fig. 1B. But here, the fluorescence image does not clearly show a Janus bead structure. Instead, some of the beads barely fluoresce, and others fluoresce green, yellow, or orange. This can be explained by a broad distribution of polymer composition in each bead. The different fluorescence colors may due to phase separated structures that are smaller than the diffraction limit of light. Different ratios and thus different intensities of green and red fluorescence then lead to different mixed colors. Thus, using PVA as emulsifier, toluene is not a good solvent to produce regular Janus beads.

Using the high boiling solvent geranyl formate does not lead to polymer beads at all. Similar to the two previous solvents, ethyl acetate and toluene, initially an oilin-water emulsion forms, but here the water evaporates
TABLE I: Average size of polymer Janus beads prepared from ethyl acetate solution at different polymer concentrations. The weight ratio of polystyrene and poly(methyl methacrylate) is 1:1. The concentration of the emulsifier (PVA, Mw: 88,000, incomplete hydrolyzed) is $1.5 \mathrm{mg} / \mathrm{mL}$. Note that the beads have a large polydispersity of around 2 , so we did not calculate the standard deviation

\begin{tabular}{cc}
\hline \hline $\begin{array}{c}\text { Total polymer } \\
\text { concentration }(\mathrm{mg} / \mathrm{mL})\end{array}$ & $\begin{array}{c}\text { diameter } \\
(\mu \mathrm{m})\end{array}$ \\
\hline 0.3 & 1.2 \\
0.6 & 1.4 \\
1.0 & 1.8 \\
1.5 & 2.2 \\
2.0 & 4.7 \\
3.0 & 6.5 \\
\hline \hline
\end{tabular}

first. Thus, during evaporation, the organic solvent becomes the continuous phase, and a 2-D bubble structure is formed in which the polymer film contains holes of various sizes. The phase separation is presumed to be below the diffraction limit of light, because no clear contrast in the fluorescence image can be seen.

Based on these results, all further experiments were performed with ethyl acetate as the organic solvent. Here, the effects of polymer concentration and polymer ratio can be summarized by using PVA as emulsifier. Finally, the effect of the emulsifier will be presented.

\section{B. Effect of polymer concentration}

The effect of polymer concentration on the size of the Janus beads for 1:1 mixtures of PS and PMMA is summarized in Table I. As expected, higher polymer concentration leads to larger bead diameters, but the large variety of bead diameters does not allow for a detailed analysis of polydispersity and standard deviation of bead diamteres, if the increase in diameter is linear with increasing concentration. At a concentration of $0.3 \mathrm{mg} / \mathrm{mL}$, the average diameter is around $1.2 \mu \mathrm{m}$, which increases to $6.5 \mu \mathrm{m}$ at a concentration of $3 \mathrm{mg} / \mathrm{mL}$. Larger concentrations give rise to samples that include large beads with a broad size distribution [19]. Samples that are summarized in Table I contain a small amount (0.12 wt\%) of poly(vinylalcohol) as an emulsifier. The shape and the internal phase separated structure of the particles depends on the interfacial free energies of both polymers with the aqueous phase (PS/water, and PMMA/water) and between each other (PS/PMMA).

Thus, even if the volume ratio of the two polymers is unity, it does not imply that always Janus beads will be formed. It has been reported by Okubo et al. that toluene solutions of PS and PMMA have interfacial tensions of $\gamma_{\mathrm{PS}}=34.4 \mathrm{mN} / \mathrm{m}$ and $\gamma_{\mathrm{PMMA}}=18.7 \mathrm{mN} / \mathrm{m}$, respectively, which is roughly half of $\gamma_{\mathrm{PS}}$ [12]. Adding an amphiphile, SDS, the interfacial tension decreases and at a SDS concentration of $6.7 \mathrm{mg} / \mathrm{mL}, \gamma_{\mathrm{PS}}$ and $\gamma_{\mathrm{PMMA}}$ drop to $4.4 \mathrm{mN} / \mathrm{m}$ and $3.8 \mathrm{mN} / \mathrm{m}$, respectively. The ratio between $\gamma_{\mathrm{PS}}$ and $\gamma_{\mathrm{PMM}}$ thus drops from 1.84 without SDS to 1.15 with SDS is added. This decrease of inter- 

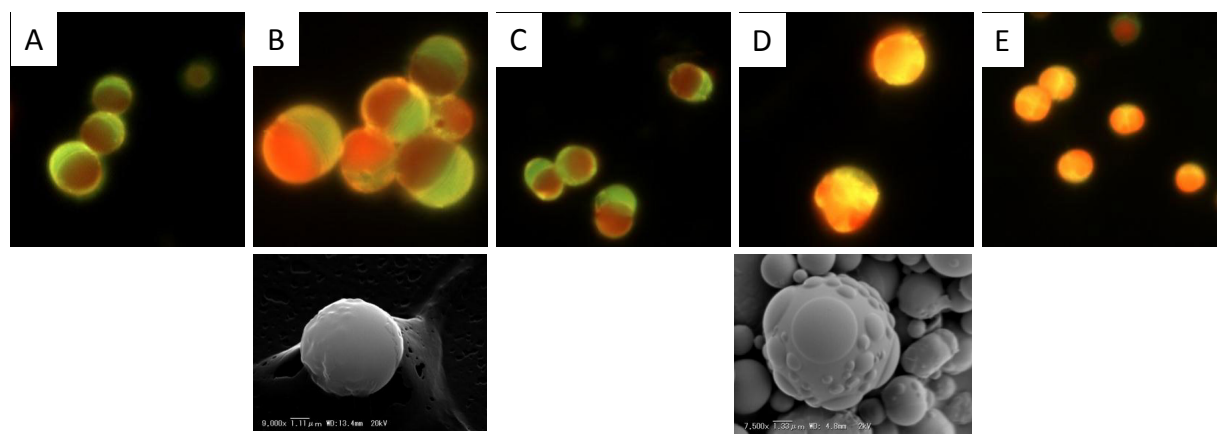

FIG. 2: Fluorescence microscope images of structures obtained from emulsions using ethyl acetate as organic solvent. The weight ratio of PS and PMMA is 1:1 with a total concentration of $3 \mathrm{mg} / \mathrm{mL}$. The aqueous phase contains poly(vinylalcohol) in different concentrations. A: $0.8 \mathrm{mg} / \mathrm{mL}$; B: $1.2 \mathrm{mg} / \mathrm{mL}$; $: 1.6 \mathrm{mg} / \mathrm{mL}$; D: $2.0 \mathrm{mg} / \mathrm{mL}$ E: $4.0 \mathrm{mg} / \mathrm{mL}$. The width of each fluorescence image is $50 \mu \mathrm{m} \times 50 \mu \mathrm{m}$. The two pictures in the bottom row are scanning electron microscope images. The boundary between the two polymer phases in the true Janus beads in (B) cannot be seen on the surface of the beads, but other phase separated structures, like the island morphology in (D) lead to a corrugation of the beads surface.

facial tension leads to a change of the phase separated structure from acorn-like (non-spherical particles with a curved polymer-polymer interface) to Janus-like, similar to the observed effect.

\section{Effect of PVA concentration}

The concentration dependence of the polymeric emulsifier, poly(vinyl alcohol), on the morphology of polymer beads was investigated, even though Okubo et al. have not observed Janus beads formed from PS/PMMA/PVA mixtures prepared from a toluene oil phase. However, spherical beads for all PVA concentrations by using ethyl acetate as oil phase are presented in Fig. 2. But similar to the observations by Okubo et al. [7], with increasing emulsifier concentration, the phase separated structure changes from acorn-like (at $0.8 \mathrm{mg} / \mathrm{mL}$ PVA) to Januslike (at $1.6 \mathrm{mg} / \mathrm{mL}$ ). Interestingly, higher PVA concentrations than $1.6 \mathrm{mg} / \mathrm{mL}$ lead to a completely different morphology: at PVA concentrations of $2 \mathrm{mg} / \mathrm{mL}$ and $4 \mathrm{mg} / \mathrm{mL}$ the former single PS phase disintegrates, leading to PMMA beads with incorporated PS islands. The reason for this drastic morphological change is not understood.

\section{Effect of the PS : PMMA ratio}

The next obvious parameter that also influences the morphology of the beads is the fraction ratio of the polymers. It can be expected that Janus-type beads are formed when using equal ratios of the two polymers. By changing their ratio, we could produce eye-like structures, at a ratio of PMMA/PS of 9:1. Here, a small sphere of PS is non-centrosymetrically embedded in a PMMA sphere. On the other hand, increasing the PS/PMMA ratio above unity, leads to PS spheres that are covered with a PMMA layer as can be seen in the fluorescence images in Fig. 3.

The question, if the PS sphere in the 9:1 mixture and the PS hemisphere in the 1:1 mixture are also covered with a PMMA layer will be addressed in the Raman spec-

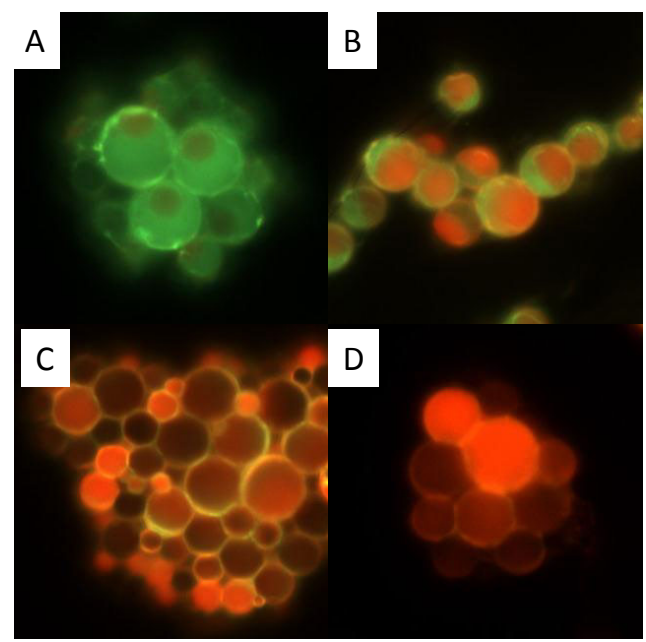

FIG. 3: Fluorescence images of Janus beads obtained from ethyl acetate emulsions with different ratios of polymer. The total polymer concentration is $3 \mathrm{mg} / \mathrm{mL}$. The ratio between PMMA and PS is 9:1 (A); 5:5 (B); 3:7 (C); 1:9 (D). The images have a width of $50 \mu \mathrm{m}$.

troscopy section of this paper. Fluorescence images, as shown here cannot fully answer this question.

\section{E. Screening of emulsifiers}

The interfacial tension of the oil phase strongly influences the overall shape of the particles, and thus the effect of various polymeric emulsifiers were tested, including many natural, carbon-neutral ones. In Fig. 4 the results obtained by using a cationic, an anionic and several neutral synthetic and natural polymers are summarized.

Chitosan is a polymer derived from the hydrolysis of chitin, poly(N-acetyl glucosamine). It contains amino groups that can be easily protonated in aqueous solution. Also, those amino groups are hydrogen-bond donors, and it has been reported that polyesters and polyamides show strong interaction with chitosan [20]. Also, the cationic 


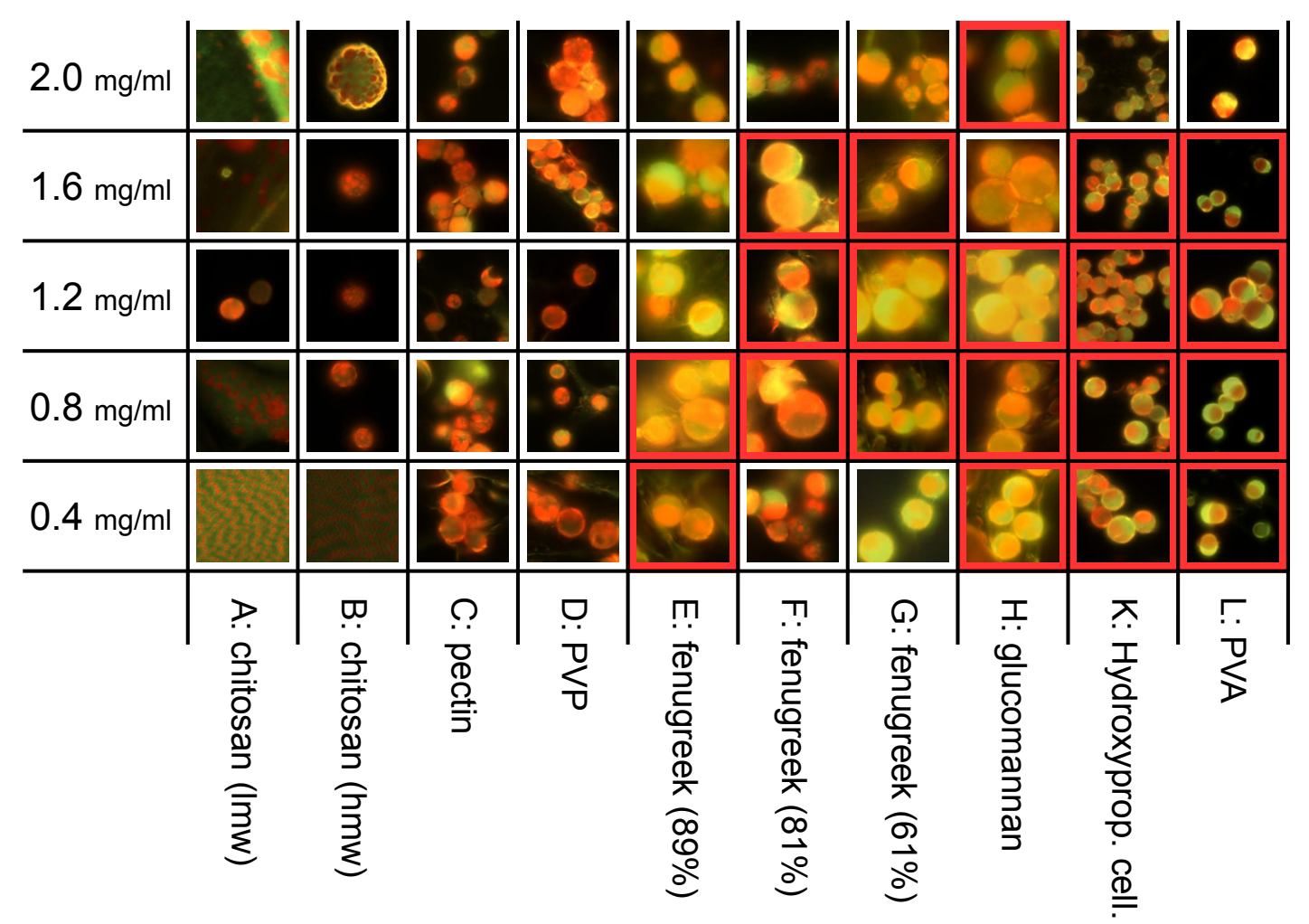

FIG. 4: Fluorescence microscope images of beads prepared from ethyl acetate containing $1.5 \mathrm{mg} / \mathrm{mL} \mathrm{PS}$ and $1.5 \mathrm{mg} / \mathrm{mL}$ PMMA. The aqueous phase contains different emulsifiers at various concentrations. A: low molecular weight chitosan; B: high molecular weight chitosan; C: pectin; D: poly(vinyl pyrrolidone); E: fenugreek gum (galactomannan 89\%); F: fenugreek gum (galactomannan 81\%); G: fenugreek gum (galactomannan 61\%); H: glucomannan; K: hydroxypropyl cellulose; L: poly(vinyl alcohol). The red rectangles indicate the mixtures that produce Janus particles. The width of the images are $50 \mu \mathrm{m}$.

chitosan can act as a surface active agent for the slightly anionic silicate glass substrate. The strong interaction between chitosan and PMMA weakens the interactions with PS, while acting as a compatibilizer for glass. So, instead of Janus beads, the PMMA forms a film on the glass substrate that is covered with PS mesas of different diameters, but similar height.

Pectins are a class of anionic heteropolysaccharides that contain a poly(galacturonic acid) backbone. Depending on the natural origin, the backbone is decorated with other saccharide groups. Since both, glass substrate and pectin, have a negative charge, their interaction is low and pectin does not act as a compatibilizer for PMMA and glass. As shown in Fig. 4, beads are produced, but the morphology is not Janus-or acorn-like. The synthetic and non-toxic poly(vinylpyrrolidone) (PVP), which had been used as synthetic blood plasma substitute, also does not produce Janus particles. Fenugreek gum is a neutral mannan polymer with galactose residues obtained from fenugreek plant. It is used as a food additive. Various sources of that polysaccharide that differ in the amount of galactose residues were screened, and most fenugreek gums produce Janus beads at concentrations between $0.4 \mathrm{mg} / \mathrm{mL}$ to $1.6 \mathrm{mg} / \mathrm{mL}$ was observed. Lower galactose content (shown in the brackets in Fig. 4) seems to push up the concentration range for the formation of Janus beads. Also, glucomannan, which contains glucose residues on a mannan polymer backbone, yields Janus beads. One interesting finding is that the beads size by using these natural polymannans is very large, typically more than $10 \mu \mathrm{m}$.

The semisynthetic hydroxypropyl cellulose has a similar effect as the other neutral polymers and produces Janus beads with typical diameters of less than $10 \mu \mathrm{m}$. The last column in Fig. 4 is poly(vinyl alcohol) that also produces Janus beads over a wide concentration range.

In summary, we could show that neutral polymers can be used for Janus beads preparation and that the type of polymer effects the resulting bead size. Here, PVA is the emulsifier of choice for small beads yielding diameters down to $1 \mu \mathrm{m}$.

\section{F. Raman imaging}

As already mentioned, interfacial tension is an important factor that determines the phase separated structure and thus Janus bead formation. Even though optical microscopy indicates the formation of two hemispheres of each polymer in the case of Janus beads, it is difficult to determine whether the hemisphere of polymer $\mathrm{A}$ is coated with a thin layer of polymer B or vice versa.

Raman spectra of PS and PMMA are well investigated and their Raman signals can be clearly distinguishable from each other [21]. Thus confocal Raman imaging was used to analyze individual polymer particles.

Figure 5 shows experimental results obtained with the above described confocal Raman microscopic setup. Light microscope images in the $x-y$ plane of two PS/PMMA 

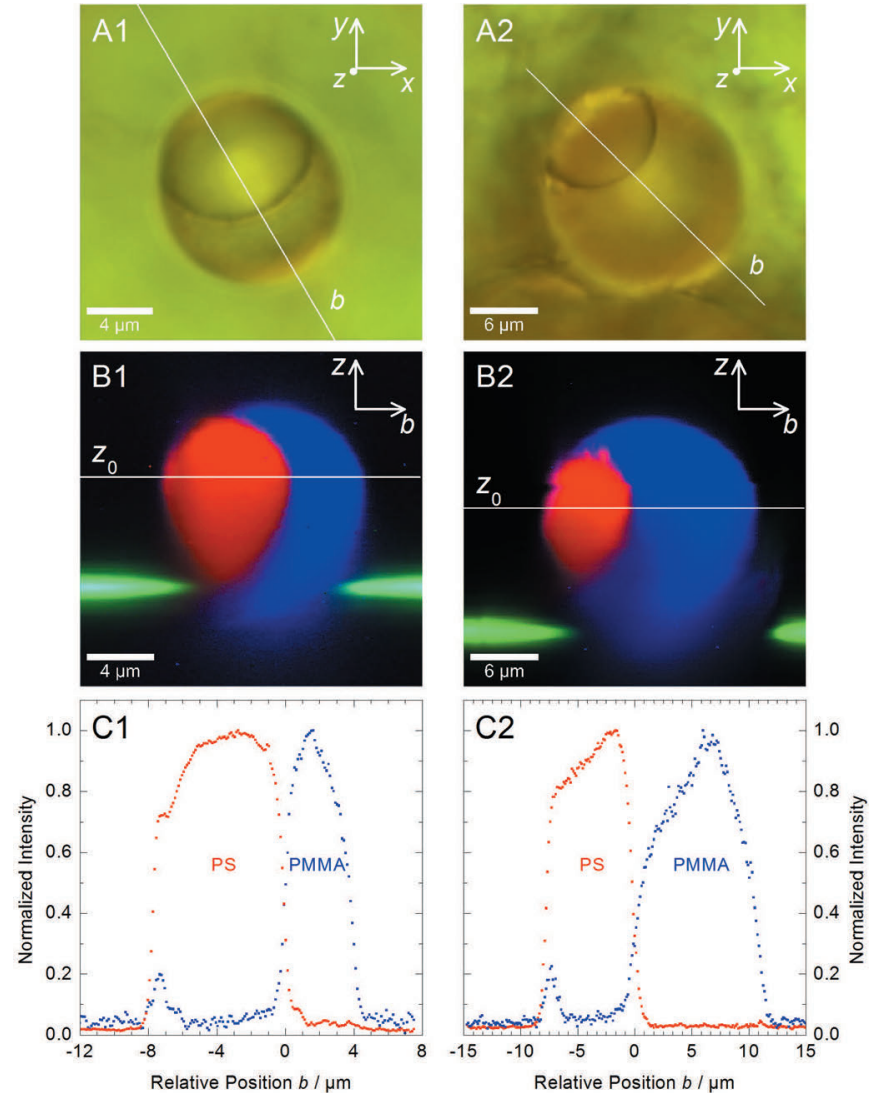

FIG. 5: Light microscope image of a Janus bead with 1:1 ratio of PS and PMMA (A1) and an 'eye' bead with a 1:9 ratio (A2). The white line $\mathrm{b}$ indicates the plane in which the 2D Raman images were taken. False color 2-D Raman image of the Janus bead (B1) and the 'eye' bead (B2). Red, blue and green denotes the intensity of the PS, PMMA and Sisubstrate signal, respectively. At $z_{0}$ the intensity of the PS and PMMA signal were extract from the 2-D Raman images. The maximum intensity for PS and PMMA was normalized to one, respectively. These intensities are plotted versus the relative position $\mathrm{b}$ for the Janus bead ( $\mathrm{C} 1)$ and the 'eye' bead (C2).

beads on a Si-substrate are presented in Fig. 5A. The Janus bead (Fig. 5A1) is a 1:1 mixture and the 'eye' bead (Fig. 5A2) is a 1:9 mixture.

In order to measure the polymer distribution across a bead, 2-D Raman images were taken in the $b-z$ plane and are presented as false color images in Fig. 5B. Each pixel in the images corresponds to one Raman spectrum. A discrimination of the Raman signals of PS and PMMA is necessary to clearly separate the PS/PMMA phase. Thus a background subtraction and a so called basis analysis were performed for each Raman spectrum. The procedure is described in detail in Ref. [16]. For the basis analysis reference spectra of PS and PMMA are extracted from the images. These spectra show all Raman signals of each polymer in the spectral region under study [21] and are used to compare each Raman spectrum of the 2-D Raman image with the above mentioned reference spectra.

In Figs. 5B1 and B2 green, red and blue color was used for the Raman signals of the Si-substrate, PS and PMMA, respectively. The signal of the Si-substrate is diminished directly below the beads. This is due to a beam displacement and a lensing effect of the green excitation light as the spherical bead acts as a microscopic ball lens. However, this effect is not crucial for the chemical analysis of the beads themselves.

The optical micrograph (Fig. 5A1) and the 2-D Raman image (Fig. 5B1) show that the 1:1 bead is not strictly a Janus bead, but more of an acorn-type with a nearly spherical PS phase. However, for the question if the PS phase is covered with a thin PMMA layer, this deviation from the ideal Janus shape is not crucial.

The 'eye' bead that formed from the 1:9 mixture also shows a nearly spherical minor PS phase in the Raman image (Fig. 5B2). Both Raman images were used to determine the chemical composition along a line that roughly cuts through the center of the PS phase. This line is perpendicular to the $z$-axis and follows the line $b$ of Figs. 5A1 and $5 \mathrm{~A} 2$, respectively. At $z=z_{0}$ it intersects the beads at the location of a maximum thickness of the PS phase. Within $z=z_{0} \pm 0.5 \mu \mathrm{m}$ the intensity of the PS and PMMA Raman signals were taken from the 2-D Raman images. Based on the above mentioned step size of $0.1 \mu \mathrm{m}$ for the generated Raman images, each measurement point is a signal of 10 Raman spectra and the maximum signal in each plot is normalized to one. The data are plotted versus the relative position $b$ and are presented in Fig. $5 \mathrm{C} 1$ (Janus bead) and Fig 5C2 ('eye' bead).

First, it has to be noted that PS and PMMA are hardly miscible. The spectral intensity in the PS phase shows low intensity of PMMA and vice versa with a sharp interface. At the relative position $b \approx-7.5 \mu \mathrm{m}$ the surface of PS on the left side of the beads shows the presence of a small PMMA signal. Artifacts like an increase of a Raman signal at the edge of a polymer/polymer interface was presented and described by Everall [22]. However, no signal of PS can be found at the opposite site of the beads at the surface of the PMMA phase. So an artifact like the collection of Raman signals due to a waveguide effect [21] seems unlikely, but is still under investigation. This small increase of the PMMA signal points to a thin skin layer of PMMA on the outer side of the PS phase.

\section{SUMMARY}

The synthesis of Janus-type polymer particles of polystyrene and poly(methyl methacrylate) was presented. Here, the environmentally friendly ethyl acetate was used as the oil phase, and natural, semisynthetic, or synthetic polymeric as emulsifiers in the water phase of an oil-in-water emulsion. The size of the particles can be controlled by polymer concentration, their shape by the weight ratio of the two polymers. For the preparation of Janus beads the kind of emulsifier is crucial, and neutral poly(vinyl alcohol) and poly(mannan)s are best suited.

Raman spectroscopy was used as a direct nondestructive optical method to investigate the inner structure of two well phase separated polymer beads. 2-D confocal Raman images show a sharp interface of the polymers. The Raman signal across the beads show an increase of the PMMA signal at the outer side of the PS phase indicating a thin skin layer of PMMA on PS. 


\section{Acknowledgments}

This work was supported by Grant-in-Aid for Scientific Research on Innovative Areas of MEXT KAKENHI. Ad- ditionally, the authors gratefully acknowledge the financial support for the "Centre for Innovation Competence" innoFSPEC of the German BMBF Program Unternehmen Region (grant no. 03Z2AN12).
[1] C. Casagrande, P. Fabre, E. Raphael, and M. Veyssie, Europhys. Lett. 9, 251 (1989).

[2] J. M. di Meglio and E. Raphael, J. Colloid Interface Sci. 136, 581 (1990).

[3] T. Ondarcuhu, P. Fabre, E. Raphael and M. Veyssie, J. Phys. Paris 51, 1527 (1990).

[4] A. Walther and M. Hoffmann, Angew. Chem. Int. Ed. 47, 711 (2008).

[5] K.-H. Roh, M. Yoshida, and J. Lahann, Langmuir 23, 5683 (2007).

[6] B. H. McNaughton, R. R. Agayan, J. X. Wang, and R. Kopelman, Sens. Act. B: Chemical 121, 330 (2007).

[7] M. Okubo, A. Yamada, and T. Matsumoto, J. Polym. Sci., Polym. Chem. Ed. 16, 3219 (1980).

[8] C. S. Winzor and D. C. Sundberg, Polymer 33, 4269 (1992).

[9] P. Rajatapiti, V. L. Dimonie, M. S. El-Aasser, and M. S. Vratsanos, J. Appl. Polym. Sci. 63, 205 (1997).

[10] M. Okubo, T. Yamashita, H. Minami, and Y. Konishi, Colloid Polym. Sci. 276, 887 (1998).

[11] M. Okubo, N. Saito, and T. Fujibayashi, Colloid. Polym. Sci. 283, 691 (2005).

[12] N. Saito, Y. Kagiri, and M. Okubo, Langmuir 22, 9397 (2006).

[13] N. Saito, Y. Kagiri, and M. Okubo, Langmuir 23, 5914 (2007).
[14] O. Karthaus and Y. Kiyono, e-J. Surf. Sci. Nanotech. 4, 270 (2006).

[15] M.-C. Wu, H.-C. Liao, Y. Chou, C.-P. Hsu, W.-C. Yen, C.-M. Chuang, Y.-Y. Lin, C.-W. Chen, Y.-F. Chen, and W.-F. Su, J. Phys. Chem. 114, 10277 (2010).

[16] T. Dieing, O. Hollricher, and J. Toporski (Eds.), Confocal Raman Microscopy (Springer, Heidelberg-DordrechtLondon-NewYork, 2010), Ch. 1, pp. 1-20, Ch. 3, pp. 43-60, and Ch. 4, pp. 61-89.

[17] T. S. Tkaczyk, Field guide to microscopy, SPIE Field Guides, Vol. FG13 (The Society of Photo-Optical Instrumentation Engeneers (SPIE), Bellingham, Washington, 2010).

[18] W. M Riemenschneider and H. Bolt, Esters, Organic in Ullmann's Encyclopedia of Industrial Chemistry (WileyVCH, 2005).

[19] O. Karthaus and J. Watanabe, Extended Abstracts, The 53rd Spring Meeting, 2006, The Japan Society of Applied Physics, p. 1293 (2006).

[20] L. M. Robeson, it Polymer Blends: a comprehensive review (Carl Hanser Verlag, Munich, 2007).

[21] B. Schrader, Raman/Infrared Atlas of Organic Compounds, N-03 and N-04 (Wiley-VCH, 1989).

[22] N. J. Everall, Appl. Spectrosc. 63, 245A (2009). 